Cochrane Database of Systematic Reviews

\title{
Confusion Assessment Method for the intensive care unit (CAM-ICU) for the diagnosis of delirium in adults in critical care settings (Protocol)
}

Miranda F, Arevalo-Rodriguez I, Díaz G, Gonzalez F, Plana MN, Zamora J, Quinn TJ, Seron P

Miranda F, Arevalo-Rodriguez I, Díaz G, Gonzalez F, Plana MN, Zamora J, Quinn TJ, Seron P.

Confusion Assessment Method for the intensive care unit (CAM-ICU) for the diagnosis of delirium in adults in critical care settings.

Cochrane Database of Systematic Reviews 2018, Issue 9. Art. No.: CD013126.

DOI: 10.1002/14651858.CD013126.

www.cochranelibrary.com

Confusion Assessment Method for the intensive care unit (CAM-ICU) for the diagnosis of delirium in adults in critical care settings (Protocol)

Copyright $\odot 2018$ The Cochrane Collaboration. Published by John Wiley \& Sons, Ltd. 


\section{TABLE OF CONTENTS}

HEADER . . . . . . . . . . . . . . . . . . . . . . . . . . . . . . . . . . . . . . . 1

ABSTRACT . . . . . . . . . . . . . . . . . . . . . . . . . . . . . . . . . . . . . . . 1

BACKGROUND . . . . . . . . . . . . . . . . . . . . . . . . . . . . . . . . . . . . . . . 1

Figure 1. . . . . . . . . . . . . . . . . . . . . . . . . . . . . . . . . . . . . .

OBJECTIVES . . . . . . . . . . . . . . . . . . . . . . . . . . . . . . . . . . . . . . . . . .

METHODS . . . . . . . . . . . . . . . . . . . . . . . . . . . . . . . . . . . . . . . . . . . 4.4

ACKNOWLEDGEMENTS . . . . . . . . . . . . . . . . . . . . . . . . . . . . . . . . . . . . . . . . .

REFERENCES . . . . . . . . . . . . . . . . . . . . . . . . . . . . . . . . . . . . . . 7

APPENDICES . . . . . . . . . . . . . . . . . . . . . . . . . . . . . . . . . . . . . . . . . . . .

CONTRIBUTIONS OF AUTHORS . . . . . . . . . . . . . . . . . . . . . . . . . . . . . . . . . . . . . . . . .

DECLARATIONS OF INTEREST . . . . . . . . . . . . . . . . . . . . . . . . . . . . . . . . 20

SOURCES OF SUPPORT . . . . . . . . . . . . . . . . . . . . . . . . . . . . . . . . . . . . . . . . . 20

Confusion Assessment Method for the intensive care unit (CAM-ICU) for the diagnosis of delirium in adults in critical care settings 


\title{
Confusion Assessment Method for the intensive care unit (CAM-ICU) for the diagnosis of delirium in adults in critical care settings
}

\author{
Fabian Miranda ${ }^{1}$, Ingrid Arevalo-Rodriguez ${ }^{2,3,4}$, Gonzalo Díaz ${ }^{1}$, Francisco Gonzalez ${ }^{1}$, Maria N Plana ${ }^{5}$, Javier Zamora ${ }^{3,4,6}$, Terry J \\ Quinn $^{7}$, Pamela Seron ${ }^{8}$ \\ ${ }^{1}$ Department of Medicine, Universidad de Chile, Santiago, Chile. ${ }^{2}$ Cochrane Ecuador. Centro de Investigación en Salud Pública y \\ Epidemiología Clínica (CISPEC). Facultad de Ciencias de la Salud Eugenio Espejo, Universidad Tecnológica Equinoccial, Quito, \\ Ecuador. ${ }^{3}$ Clinical Biostatistics Unit, Hospital Universitario Ramon y Cajal (IRYCIS), Madrid, Spain. ${ }^{4}$ CIBER Epidemiology and \\ Public Health (CIBERESP), Madrid, Spain. ${ }^{5}$ Universidad Francisco de Vitoria (UFV) Madrid. CIBER Epidemiology and Public \\ Health (CIBERESP), Madrid, Spain. ${ }^{6}$ Women's Health Research Unit, Centre for Primary Care and Public Health, Queen Mary \\ University of London, London, UK. ${ }^{7}$ Institute of Cardiovascular and Medical Sciences, University of Glasgow, Glasgow, UK. ${ }^{8}$ CIGES, \\ Departamento de Medicina Interna, Facultad de Medicina, Universidad de La Frontera, Temuco, Chile
}

Contact address: Pamela Seron, CIGES, Departamento de Medicina Interna, Facultad de Medicina, Universidad de La Frontera, Montt112, 3o piso, Temuco, Araucania, 4780000, Chile. pamela.seron@ufrontera.cl.

Editorial group: Cochrane Dementia and Cognitive Improvement Group.

Publication status and date: New, published in Issue 9, 2018.

Citation: Miranda F, Arevalo-Rodriguez I, Díaz G, Gonzalez F, Plana MN, Zamora J, Quinn TJ, Seron P. Confusion Assessment Method for the intensive care unit (CAM-ICU) for the diagnosis of delirium in adults in critical care settings. Cochrane Database of Systematic Reviews 2018, Issue 9. Art. No.: CD013126. DOI: 10.1002/14651858.CD013126.

Copyright (C) 2018 The Cochrane Collaboration. Published by John Wiley \& Sons, Ltd.

\begin{abstract}
A B S T R A C T
This is a protocol for a Cochrane Review (Diagnostic test accuracy). The objectives are as follows:

To determine the diagnostic accuracy of the Confusion Assessment Method for the Intensive Care Unit (CAM-ICU) for the diagnosis of delirium in adult patients in critical care settings.
\end{abstract}

\section{B A C K G R O U N D}

Delirium is a common problem in general clinical practice and its incidence increases with age. One-third of older patients have delirium during hospitalization, with half of them presenting with it at the time of admission (Marcantonio 2017). Delirium is also a common problem in the postoperative period (Marcantonio 2012), in emergency units (Kennedy 2014), and in palliative care (Inouye 2014). Since patients in intensive care units (ICUs) have a considerable number of predisposing comorbidities (Marra 2017a), delirium is a common issue in this setting, with an estimated incidence of up to $80 \%$ (Ely 2004). The need for a useful and efficient tool for diagnosis of delirium in the ICU has become more widespread. The Confusion Assessment Method for the Intensive Care Unit (CAM-ICU) is a tool recommended by the clinical guidelines (Barr 2013), and it is widely used in research and clinical settings (Estrup 2017; Kenes 2017; Khan 2017; Singh 2018).

Delirium is associated with a variety of adverse and deleterious

Confusion Assessment Method for the intensive care unit (CAM-ICU) for the diagnosis of delirium in adults in critical care settings 
outcomes (Ely 2004; Thomason 2005) including increased length of hospital stay (Akunne 2012; Lee 2014), increased mortality up to 12 months after hospital discharge, persisting cognitive impairments at three and 12 months of follow-up and increased rates of hospital re-admissions (Gunther 2008; Tobar 2010; Witlox 2010). In addition, delirium has a substantial economic impact for patients, clinicians and healthcare facilities (McCusker 2002; Leslie 2008).

The first step in managing delirium is making a timely diagnosis; thus, active surveillance for delirium is recommended in all critically ill patients (Barr 2013; Marra 2017). Protocols that include serial measurement for delirium have reported a reduction in the incidence and duration of delirium, improvement of the functional status of patients and a reduction in coma duration, mechanical ventilation and length of hospital stay (Balas 2014; Collinsworth 2016). In addition, compliance with these protocols has been independently associated with improvements in survival (Barnes-Daly 2017).

Due to the complexity of its diagnosis, delirium remains an under-diagnosed condition, especially in patients undergoing invasive mechanical ventilation (Otter 2005; Trogrlic 2015). Factors specific to the ICU setting that may complicate the assessment of delirium include the need for sedation, the difficulty of communicating during orotracheal intubation and the mixed skills of staff, among others.

\section{Target condition being diagnosed}

Delirium (also known as acute-confusional state) is a clinical syndrome defined as a disturbance of attention and consciousness, with an acute presentation and tendency to fluctuate in severity during the course of the day (American Psychiatric Association 2013). This condition is classified into three subtypes: hyperactive, hypoactive and mixed delirium (Morandi 2009). Hyperactive delirium is characterized by increased psychomotor behavior, while in hypoactive delirium the patient shows reduced psychomotor behavior, as well as decreased alertness and apathy. Mixed delirium is characterized by alternating episodes of agitation, aggressiveness and hypoactivity (Morandi 2009). Although many clinicians consider agitation as the distinctive symptom of delirium, this increase in the psychomotor behavior represents only $25 \%$ of cases, with the other $75 \%$ having hypoactive ("quiet") conditions (Marcantonio 2017).

The pathophysiology of delirium is still insufficiently understood despite the fact that several theories have been proposed to explain its onset (Jackson 2015). Risk factors for incident delirium, which is common in patients admitted to the ICU (Van Rompaey 2009), include advanced age, cognitive decline prior to admission, visual or auditory impairment, history of alcohol abuse and previous use of benzodiazepines (Ely 2001c; Hshieh 2015). In addition, precipitating factors that are widely recognized are the use of catheters, untreated pain, psychoactive medications, sleep deprivation, severe sepsis, hypoxaemia, dehydration, hypotension, biochemical abnormalities and anemia (Morandi 2009).

While there is increasing research aimed at characterizing delirium in the pediatric population, including validation of specific diagnostic instruments (Harris 2016; Patel 2017), most of the existing knowledge comes from the adult population, especially from older adults, where delirium is extremely common (Marcantonio 2011).

\section{Index test(s)}

The CAM-ICU is a short test for the diagnosis of delirium. The CAM-ICU was developed from the longer Confusion Assessment Method (CAM) tool often used in older adults (Ely 2001a). The CAM-ICU is characterized by its rapid administration and no requirement for verbal communication from the patient, which allows it to be administered in patients undergoing invasive mechanical ventilation and orotracheal intubation (Thomason 2005). The test follows a detailed protocol; and with adequate training, it can be administered by any member of the ICU staff (Pisani 2007).

The CAM-ICU adopts a staged approach to assessment. In the first stage, the level of consciousness is assessed (using a validated sedation/level of consciousness scale), and a decision is made as to whether the patient should be further assessed or can be reevaluated later. The next step is an assessment of the content of consciousness with verification of four cardinal criteria, each of them with two levels (absent and present), used to establish the presence of delirium, namely, the presence of alterations or fluctuation (or both) in the mental status (scores as absent or present) (Ely 2016). See Figure 1. 
Figure I. Confusion Assessment Method for the ICU (CAM-ICU) flow sheet. (Ely 20 I6)

\section{Confusion Assessment Method for the ICU (CAM-ICU) Flowsheet}

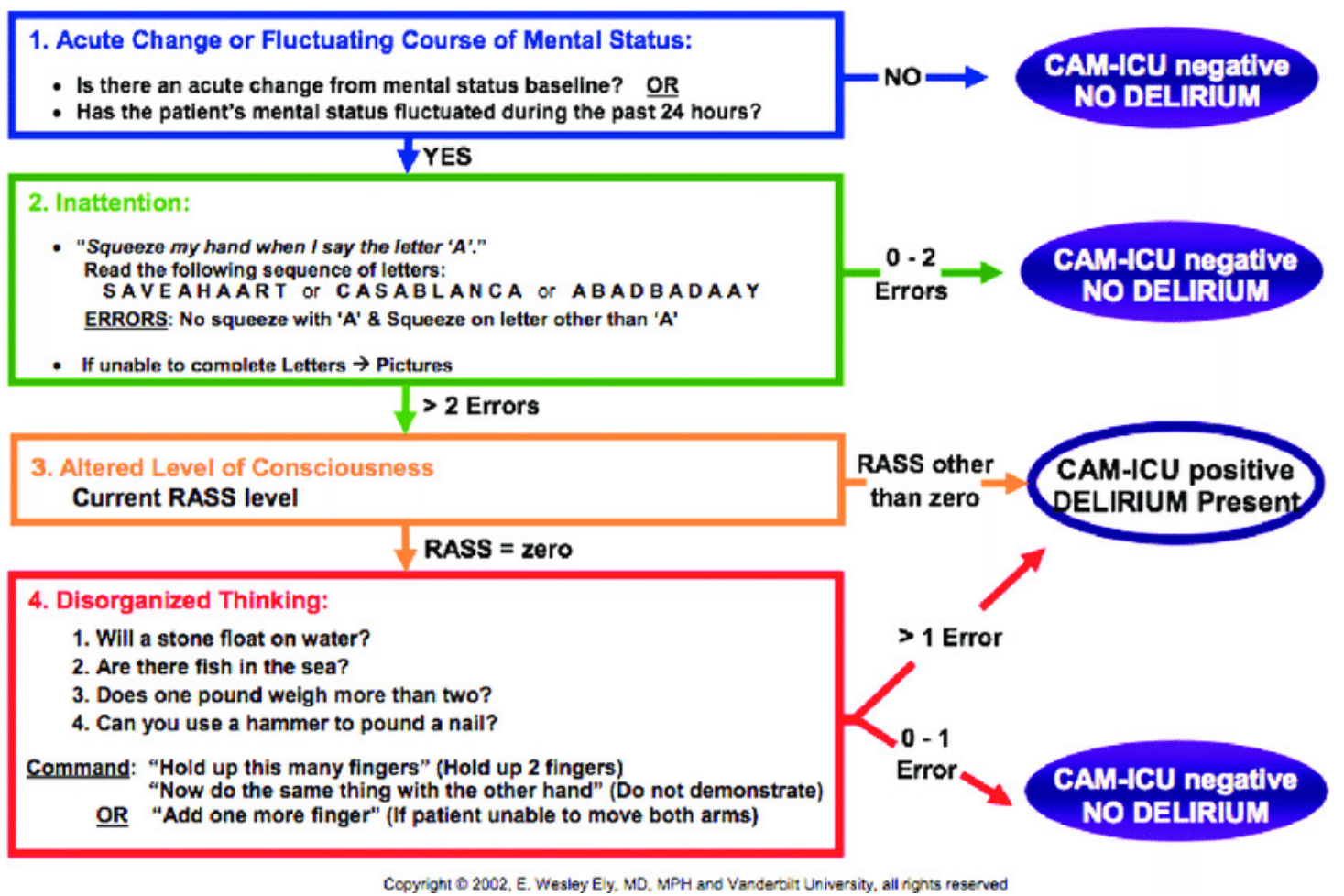

The diagnosis of delirium is based on the presence of two major criteria (i.e. acute or fluctuating onset plus lack of attention) and at least one of the minor criteria (disorganized thinking or altered consciousness level) (Gonzalez 2003). Some studies have reported adequate performance of the CAM-ICU, including an acceptable inter-rater reliability (Thomason 2005), strong correlations with clinical outcomes such as length of hospital stay and mortality (Ely 2004; Lin 2004; Thomason 2005), as well as a promising estimation of test accuracy (Boettger 2017).

\section{Clinical pathway}

In the past, a reference standard or other alternative tests were needed to confirm a suspected diagnosis of delirium (i.e. confused patient), whereas in current clinical practice, the CAM-ICU is used without the need of previous tests for diagnosis confirmation. In addition, based on the high frequency of incident delirium, the low level of clinical suspicion and the prevalence of hypoactive forms (Marcantonio 2017), all patients in the ICU are considered as being at risk of delirium and should be regularly (i.e. once per shift) assessed for this condition (Barr 2013).

The CAM-ICU is considered to be a diagnostic test because the population of patients cannot be labelled as truly asymptomatic before applying it; however, some clinicians also consider it to be as a screening tool (Wong 2010). A "positive" CAM-ICU triggers a complete clinical evaluation in search of the underlying cause (Barr 2013; Marcantonio 2017). This comprehensive assessment includes a medical history review, a complete physical examination, laboratory assays and complementary tests. Treatment of the etiology causing the delirium starts as soon as the diagnosis is confirmed. On the contrary, if the test is "negative", the CAM-ICU is repeated when a new clinical suspicion appears or according to the ICU assessment schedule (Barr 2013).

\section{Alternative test(s)}

In addition to the CAM-ICU, there are several questionnaires to assess delirium. One of the most well known is the Intensive Care Delirium Screening Checklist (ICDSC), which is based on a stratification scale for patients with delirium (Inouye 1990; Brummel 2013). The ICDSC is widely available in several languages (Ely 2001b). However, its administration is difficult in ventilated patients, it requires training to interpret findings, and requires a specific timeframe for assessment (last 24 hours), which can lead to an 
increase of false positives (Inouye 1990; Brummel 2013). Other instruments for the diagnosis of delirium include:

- the Delirium Detection Score (DDS), which is based on an instrument designed to evaluate delirium associated with alcoholic deprivation (Wong 2010);

- the Cognitive Test of Delirium (CTD), a tool that requires a considerable amount of time for its administration, with a summarized version which has not been properly validated (Wong 2010);

- the Memorial Delirium Assessment Scale (MDAS), originally designed for patients with advanced cancer and later adapted for use in critically ill patients (Immers 2005; Fadul 2007; Carvalho 2013);

- the Neelon and Champagne Confusion Scale (NEECHAM) and the Delirium Rating Scale-Revised 98 (DRSR-98) are also useful, but less widely used (Trzepacz 1994; Shi 2013).

The electroencephalogram (EEG) has also been used in the diagnosis of delirium. This method allows the observation of a slow wave pattern, which is an expression of cerebral metabolic damage that is remarkably sensitive but non-specific (Bergeron 2001; Gusmao-Flores 2012). Due to its low specificity and issues with clinical applicability, it is not considered a practical test in the assessment of critically ill patients.

\section{Rationale}

The high incidence and deleterious consequences of delirium require a timely and practical approach to diagnosis. The use of the CAM-ICU can improve diagnosis and prompt treatment, as well as reduce the undesirable consequences of this condition (Barr 2013; Serafim 2015). Unlike other tools, the CAM-ICU test has been specifically designed to assess delirium in critically ill patients (especially those intubated), and has encouraging features such as its ease of use and minimal training requirements. Therefore, it can be applied by non-medical personnel, which promotes teamwork, saves resources and avoids under-diagnosis. This tool has also been translated and adapted for use in multiple languages. The information available in the medical literature about the diagnostic accuracy of CAM-ICU is highly heterogeneous and ambiguous (Barr 2013; Estrup 2017; Kenes 2017; Khan 2017; Singh 2018); a critical and updated synthesis of the test accuracy of the CAM-ICU is needed for clinicians, policymakers and for those ICU teams who are already using the tool. The introduction of a delirium assessment program should also be considered.
To determine the diagnostic accuracy of the Confusion Assessment Method for the Intensive Care Unit (CAM-ICU) for the diagnosis of delirium in adult patients in critical care settings.

\section{Secondary objectives}

To explore potential sources of heterogeneity, as well as specific factors that may influence the accuracy of the CAM-ICU, including: characteristics of recruited patients (patients from medical versus surgical ICUs and type of ventilatory assistance); characteristics of delirium (subtype of delirium or duration of symptoms); characteristics of administration (ICU staff member performing the test); and reference standard used, among others.

\section{METHODS}

\section{Criteria for considering studies for this review}

\section{Types of studies}

We will include diagnostic studies that describe the accuracy of the Confusion Assessment Method for the Intensive Care Unit (CAM-ICU) for the diagnosis of delirium in adult patients admitted in intensive care units (ICUs). We will include studies that are published in any language. We will consider full paper articles and abstracts, published conference proceedings, and posters. However, if these selected references do not provide enough information for the assessment of methodological quality, we will classify them as 'awaiting assessment'. We will exclude case-control studies due to the high risk of bias involved in their development and in the final estimation of test accuracy (Rutjes 2005). We will exclude studies that provide insufficient information to derive a standard $2 \times 2$ table, as well as case reports.

\section{Participants}

We will include adult patients (18 years of age or older) admitted to ICUs and with suspected delirium, independent of the baseline pathology, and whether or not they are submitted to mechanical ventilation. Intensive care units are broadly defined as a hospital subsection capable of: intensive or invasive monitoring; support of airway, breathing or circulation; stabilization of acute or lifethreatening medical problems; comprehensive management of injury or illness (or both); and restoration to stable health status or comfort during end-of-life care. Patients at ICUs are a heterogeneous population, but they all share the need for close assessment and monitoring, as well as the requirement of life support (SCCM 2018). 


\section{Index tests}

We will assess the CAM-ICU tool, applied by any trained personnel, medical or non-medical (Barr 2013). We will accept those cases in which adjustments of the original test have been made to local conditions (including language translations and socio-cultural adaptations). A 'positive' CAM-ICU will be based on the presence of two major criteria (acute or fluctuating onset and lack of attention) and at least one of the minor criteria (disorganized thinking or altered consciousness level) (Gonzalez 2003), following the instructions of the original authors (Ely 2016). Since the test has two possible outcomes (positive or negative), we will not observe different and explicit cut-offs. However, it is likely that different studies could have used different implicit thresholds to determine a positive CAM-ICU test result depending on the staff training and their knowledge or experience of the target condition. This issue can be investigated as a source of heterogeneity (see Investigations of heterogeneity).

\section{Target conditions}

Delirium is a syndrome defined as a disturbance of attention and consciousness, which presents acutely and tends to fluctuate in severity during the course of the day (American Psychiatric Association 2013). Delirium is currently defined by the clinical criteria proposed by the American Psychiatric Association in the Diagnostic and Statistical Manual of Mental Disorders (DSM) (American Psychiatric Association 2013). We will include all subtypes, types and severities of delirium in our review.

\section{Reference standards}

We will use acceptable and commonly used reference standards for delirium diagnosis, according to the development and publication date of each study. We will consider the diagnostic criteria developed by the American Psychiatry Association (American Psychiatric Association 2013), considering all versions of the DSM. The DSM criteria should be applied by clinicians specialized in recognizing the signs and symptoms of delirium in the ICU.

\section{Search methods for identification of studies}

\section{Electronic searches}

We will search MEDLINE (Ovid SP, 1966 to date), Embase (Ovid SP, 1982 to date), BIOSIS (Ovid, inception to date), Science Citation Index (ISI Web of Knowledge, inception to date), PsycINFO (Ovid SP, inception to date) and LILACS (BIREME, 1982 to date). Science Citation Index includes conference abstracts in its database (see Appendix 1 for a proposed search strategy draft to be run in MEDLINE). We will design similarly structured search strategies using search terms appropriate for each database. We will use controlled vocabulary such as MeSH terms and EMTREE where appropriate.

We will not use search filters designed to retrieve diagnostic test accuracy studies as a method to restrict the search overall, since available filters have not yet proved sensitive enough for systematic review searches (Whiting 2011). However, we will adopt a "twopronged approach" as described in the Cochrane Handbook for Systematic Reviews of Diagnostic Test Accuracy (Deeks 2013; de Vet 2008). We will not apply any language restriction to the electronic searches.

\section{Searching other resources}

We will perform a search for relevant studies in the references of the included studies. We will also search the following.

- Health Technology Assessment database (HTA database) in the Cochrane Library (www.cochranelibrary.com)

- Aggressive Research Intelligence Facility ( ARIF database) ( 147.188.28.230/rmwp)

- WHO International Clinical Trials Registry Platform ( apps.who.int/trialsearch/)

- ClinicalTrials.gov ( clinicaltrials.gov)

- Websites of scientific associations such as the American Psychiatric Association, the Royal College of Psychiatrists and the Intensive Care Society, to access any annual meetings and abstracts of conference proceedings in the field.

We will also consult with experts and attempt to contact study authors when necessary to obtain additional information on potential studies.

\section{Data collection and analysis}

\section{Selection of studies}

Two review authors (FM and FG) will independently select the studies, based on the title and abstract. Once the potentially eligible articles are identified, and the complete versions of each are obtained, two review authors (FM and GD) will independently evaluate each study for inclusion or exclusion. We will resolve disagreements through discussion. We will present the study selection process in a PRISMA flow diagram.

\section{Data extraction and management}

We will extract data on study characteristics to a study-specific pro-forma (Appendix 2), and we will include data on assessment of quality and investigation of heterogeneity. It will include the general information of each study, epidemiological data of included patients, details of test administration and details of the delirium 
diagnosis process. This process will be carried out by two independent review authors (FM and FG). We will dichotomize the CAM-ICU results and cross-tabulate in $2 \times 2$ tables the index test results (positive or negative) against the reference standard (positive or negative). We will use Review Manager 5 to describe all these findings.

\section{Assessment of methodological quality}

We will assess the methodological quality of each study by using the Quality Assessment of Diagnostic Accuracy Studies (QUADAS2) (Whiting 2011a). This tool is made up of four domains: patient selection, index test, reference standard and patient flow (see Appendix 3). Each domain is assessed in terms of risk of bias, and the first three domains are also considered in terms of applicability. The potential risk of bias associated with each domain is rated as high, low, or uncertain. Two review authors (FM and GD) will evaluate the articles independently. They will discuss and reach consensus on the quality of the included studies and problems of applicability of each study. We will resolve disagreements through discussion.

\section{Statistical analysis and data synthesis}

A template for creating $2 \times 2$ tables describing true positive (TP), false positive (FP), false negative (FN) and true negative (TN) results will be applied for each included study. From this, we will calculate sensitivity and specificity, together with their $95 \%$ confidence intervals, at the individual study level. We will present individual study results graphically by plotting estimates of sensitivities and specificities in forest plots. We will enter data into Review Manager 5 software and check it for accuracy (Review Manager 2014).

We will perform the meta-analyses according to the guidelines described in Chapter 10 of the Cochrane Handbook for Systematic Reviews of Diagnostic Test Accuracy (Macaskill 2010). We will estimate the parameters of the bivariate model (Reitsma 2005; Chu 2006), using the meqrlogit command in STATA 15. We will transfer these parameters from the bivariate model into Review Manager 5 to produce a summary receiver operating characteristic (ROC) curve and a summary point representing summary sensitivity and specificity, along with $95 \%$ confidence ellipse and a $95 \%$ prediction ellipse. For investigation of sources of heterogeneity, we will introduce covariates in the fixed part of the model to check whether sensitivity, specificity, or both, depend on selected covariates. We will use likelihood ratio tests to compare models with and without the covariate, one at each time.

If the review includes a limited number of studies or if the studies showed zero false negatives or false positives that make the hierarchical model difficult to fit (i.e. non-convergence), we will simplify model parameterization. We will assume no correlation between indices and will simplify the model to two univariate fixed- effect or random-effects models (Takwoingi Y 2015), depending on variability in sensitivity or specificity (or both) between studies observed in the plots.

\section{Investigations of heterogeneity}

We will initially investigate heterogeneity by visually inspecting the forest plots for sensitivity and specificity, and by evaluating the individual results of the studies in the ROC space, with the purpose of examining the variability between studies and the presence of a correlation between both indices (threshold effect). The heterogeneity will be quantified by the variance of logit sensitivity and specificity estimated by the model.

If enough data are available, we will explore possible sources of heterogeneity through subgroup analyses. Anticipated sources of heterogeneity are: medical versus surgical ICU patients; type of ventilatory assistance (mechanical, spontaneous); subtype of delirium (hyperactive, hypoactive, mixed); severity of delirium; staff member performing the test (nurses, general physicians, residents/ fellows, other healthcare professionals); reference standard used; and if the tool was adapted or not.

Assuming that an adequate number of studies report study-level covariates, we will investigate the effect of these by including each of these factors as covariates in the bivariate regression model. We will assess the model fit by using likelihood ratio tests. This will allow us to test whether sensitivity or specificity, or both, differed in subgroups of studies defined by these covariates.

\section{Sensitivity analyses}

We will evaluate the robustness of the meta-analysis by conducting a sensitivity analysis that excludes studies that are considered to be at high risk of bias and high concerns in applicability. Our primary analysis will include all studies, while the sensitivity analysis will exclude studies at high risk of bias or with significant concerns about applicability in at least one domain. We will report the results of the sensitivity analysis for each domain, using a summary table.

\section{Assessment of reporting bias}

We will not investigate reporting bias because of current uncertainty about how it operates in diagnostic test accuracy studies and the interpretation of existing analytical tools such as funnel plots.

\section{ACKNOWLEDGEMENTS}

We acknowledge Anna Noel-Storr for her help in designing the search strategy, and the editorial staff at Cochrane Dementia and Cognitive Improvement. Ingrid Arevalo-Rodriguez is funded by a Sara Borrell contract from the Instituto de Salud Carlos III (CD17/ 
00219; Acción Estrategica en Salud 2013-2016, co-funded by European Social Fund 2014 to 2020, "Investing in your future").

\section{R E F E R E N C E S}

\section{Additional references}

\section{Akunne 2012}

Akunne A, Murthy L, Young J. Cost-effectiveness of multicomponent interventions to prevent delirium in older people admitted to medical wards. Age and Ageing 2012;41 (3):285-91. [PUBMED: 22282171]

\section{American Psychiatric Association 2013}

American Psychiatric Association. Diagnostic and statistical manual of mental disorders. 5th Edition. Washington, DC: APA, 2013.

Balas 2014

Balas MC, Vasilevskis EE, Olsen KM, Schmid KK, Shostrom V, Cohen MZ, et al. Effectiveness and safety of the awakening and breathing coordination, delirium monitoring/management, and early exercise/mobility bundle. Critical Care Medicine 2014;42(5):1024-36. [PUBMED: 24394627]

\section{Barnes-Daly 2017}

Barnes-Daly MA, Phillips G, Ely EW. Improving hospital survival and reducing brain dysfunction at seven California Community Hospitals: Implementing PAD Guidelines Via the ABCDEF Bundle in 6,064 Patients. Critical Care Medicine 2017;45(2):171-8. [PUBMED: 27861180]

\section{Barr 2013}

Barr J, Fraser GL, Puntillo K, Ely EW, Gelinas C, Dasta JF, et al. Clinical practice guidelines for the management of pain, agitation, and delirium in adult patients in the intensive care unit. Critical Care Medicine 2013;41(1): 263-306. [PUBMED: 23269131]

\section{Bergeron 2001}

Bergeron N, Dubois MJ, Dumont M, Dial S, Skrobik Y. Intensive Care Delirium Screening Checklist: evaluation of a new screening tool. Intensive Care Medicine 2001;27(5): 859-64. [PUBMED: 11430542]

\section{Boettger 2017}

Boettger S, Nuñez DG, Meyer R, Richter A, Fernandez SF, Rudiger A, et al. Delirium in the intensive care setting: A reevaluation of the validity of the CAM-ICU and ICDSC versus the DSM-IV-TR in determining a diagnosis of delirium as part of the daily clinical routine. Palliative \& Supportive Care 2017;15(6):675-83. [PUBMED: 28173895]

\section{Brummel 2013}

Brummel NE, Vasilevskis EE, Han JH, Boehm L, Pun BT, Ely EW. Implementing delirium screening in the ICU: secrets to success. Critical Care Medicine 2013;41(9): 2196-208. [PUBMED: 23896832]

\section{Carvalho 2013}

Carvalho JP, de Almeida AR, Gusmao-Flores D. Delirium rating scales in critically ill patients: a systematic literature review. Revista Brasileira de Terapia Intensiva 2013;25(2): 148-54. [PUBMED: 23917980]

Chu 2006

Chu H, Cole SR. Bivariate meta-analysis of sensitivity and specificity with sparse data: a generalized linear mixed model approach. Journal of Clinical Epidemiology 2006;59 (12):1331-2. [PUBMED: 17098577]

\section{Collinsworth 2016}

Collinsworth AW, Priest EL, Campbell CR, Vasilevskis EE, Masica AL. A review of multifaceted care approaches for the prevention and mitigation of delirium in Intensive Care Units. Journal of Intensive Care Medicine 2016;31(2): 127-41. [PUBMED: 25348864]

de Vet 2008

de Vet HCW, Eisinga A, Riphagen II, Aertgeerts B, Pewsner D. Chapter 7: Searching for Studies. Cochrane Handbook for Systematic Reviews of Diagnostic Test Accuracy. 0.4. The Cochrane Collaboration, 2008.

\section{Deeks 2013}

Deeks JJ, Bossuyt PM, Gatsonis C (editors). Cochrane Handbook for Systematic Reviews of Diagnostic Test Accuracy Version 1.0.0. The Cochrane Collaboration, 2013. Available from: http://srdta.cochrane.org/.

Ely 2001a

Ely EW, Gautam S, Margolin R, Francis J, May L, Speroff $\mathrm{T}$, et al. The impact of delirium in the intensive care unit on hospital length of stay. Intensive Care Medicine 2001;27 (12):1892-900. [PUBMED: 11797025]

\section{Ely 2001b}

Ely EW, Margolin R, Francis J, May L, Truman B, Dittus $\mathrm{R}$, et al. Evaluation of delirium in critically ill patients: validation of the Confusion Assessment Method for the Intensive Care Unit (CAM-ICU). Critical Care Medicine 2001;29(7):1370-9. [PUBMED: 11445689]

Ely 2001c

Ely EW, Siegel MD, Inouye SK. Delirium in the intensive care unit: an under-recognized syndrome of organ dysfunction. Seminars in Respiratory and Critical Care Medicine 2001;22(2):115-26. [PUBMED: 16088667]

\section{Ely 2004}

Ely EW, Shintani A, Truman B, Speroff T, Gordon SM, Harrell FE Jr, et al. Delirium as a predictor of mortality in mechanically ventilated patients in the intensive care unit. Journal of the American Medical Association 2004;291(14): 1753-62. [PUBMED: 15082703] 
Ely 2016

Ely EW. Confusion Assessment Method for the ICU (CAM-ICU), The Complete Training Manual. Confusion Assessment Method for the ICU (CAM-ICU), The Complete Training Manual. Revised. Nashville: Vanderbilt University, 2016:1-32.

Estrup 2017

Estrup S, Kjer CKW, Poulsen LM, Gøgenur I, Mathiesen O. Delirium and effect of circadian light in the intensive care unit: a retrospective cohort study. Acta Anaesthesiologica Scandinavica 2018;62(3):367-75. [PUBMED: 29148046]

\section{Fadul 2007}

Fadul N, Kaur G, Zhang T, Palmer J L, Bruera E. Evaluation of the Memorial Delirium Assessment Scale (MDAS) for the screening of delirium by means of simulated cases by palliative care health professionals. Supportive Care in Cancer 2007;15(11):1271-6. [PUBMED: 17387520 ]

\section{Gonzalez 2003}

Gonzalez M, de Pablo J, Valdes M. Delirium: the clinical confusion [Delirium: la confusión de los clínicos]. Revista Medica de Chile 2003;131(9):1051-60. [PUBMED: 4635594]

\section{Gunther 2008}

Gunther ML, Morandi A, Ely EW. Pathophysiology of delirium in the intensive care unit. Critical Care Clinics 2008;24(1):45-65. [PUBMED: 18241778]

Gusmao-Flores 2012

Gusmao-Flores D, Salluh JI, Chalhub RA, Quarantini LC. The confusion assessment method for the intensive care unit (CAM-ICU) and intensive care delirium screening checklist (ICDSC) for the diagnosis of delirium: a systematic review and meta-analysis of clinical studies. Critical Care 2012;16 (4):R115. [PUBMED: 22759376]

\section{Harris 2016}

Harris J, Ramelet AS, van Dijk M, Pokorna P, Wielenga J, Tume L, et al. Clinical recommendations for pain, sedation, withdrawal and delirium assessment in critically ill infants and children: an ESPNIC position statement for healthcare professionals. Intensive Care Medicine 2016;42(6):972-86. [PUBMED: 27084344]

\section{Hshieh 2015}

Hshieh TT, Yue J, Oh E, Puelle M, Dowal S, Travison T, et al. Effectiveness of multicomponent nonpharmacological delirium interventions: a meta-analysis. Journal of the American Medical Association Internal Medicine 2015;175 (4):512-20. [PUBMED: 25643002]

Immers 2005

Immers HE, Schuurmans MJ, van de Bijl JJ. Recognition of delirium in ICU patients: a diagnostic study of the NEECHAM confusion scale in ICU patients. BioMed Central Nursing 2005;13:4-7. [PUBMED: 16351715]

\section{Inouye 1990}

Inouye SK, van Dyck CH, Alessi CA, Balkin S, Siegal AP, Horwitz RI. Clarifying confusion: the confusion assessment method. A new method for detection of delirium. Annals of Internal Medicine 1990;113(12):941-8. [PUBMED: 2240918]

Inouye 2014

Inouye SK, Westendorp RG, Saczynski JS. Delirium in elderly people. Lancet 2014;383(9920):911-22. [PUBMED: 23992774]

\section{Jackson 2015}

Jackson P, Khan A. Delirium in critically ill patients. Critical Care Clinics 2015;31(3):589-603. [PUBMED: 26118922]

\section{Kenes 2017}

Kenes MT, Stollings JL, Wang L, Girard TD, Ely EW, Pandharipande PP. Persistence of delirium after cessation of sedatives and analgesics and impact on clinical outcomes in critically ill patients. Pharmacotherapy 2017;37(11): 1357-65. [PUBMED: 28845902]

\section{Kennedy 2014}

Kennedy M, Enander RA, Tadiri SP, Wolfe RE, Shapiro NI, Marcantonio ER. Delirium risk prediction, healthcare use and mortality of elderly adults in the emergency department. Journal of the American Geriatrics Society 2014; 62(3):462-9. [PUBMED: 24512171 ]

\section{Khan 2017}

Khan SH, Wang S, Harrawood A, Martinez S, Heiderscheit A, Chlan L, et al. Decreasing Delirium through Music (DDM) in critically ill, mechanically ventilated patients in the intensive care unit: study protocol for a pilot randomized controlled trial. Trials 2017;18(1):574. [PUBMED: 29187230]

\section{Lee 2014}

Lee E, Kim J. Cost-benefit analysis of a delirium prevention strategy in the intensive care unit. Nursing in Critical Care 2016;21(6):367-73. [PUBMED: 25351583]

\section{Leslie 2008}

Leslie DL, Marcantonio ER, Zhang Y, Leo-Summers L, Inouye SK. One-year health care costs associated with delirium in the elderly population. Archives of Internal Medicine 2008;168(1):27-32. [PUBMED: 18195192]

\section{Lin 2004}

Lin SM, Liu CY, Wang CH, Lin HC, Huang CD, Huang PY, et al. The impact of delirium on the survival of mechanically ventilated patients. Critical Care Medicine 2004;32(11):2254-9. [PUBMED: 15640638]

\section{Macaskill 2010}

Macaskill P, Gatsonis C, Deeks JJ, Harbord RM, Takwoing Y. Chapter 10: Analysing and Presenting Results. Deeks JJ, Bossuyt PM, Gatsonis C (editors), Cochrane Handbook for Systematic Reviews of Diagnostic Test Accuracy Version 1.0. The Cochrane Collaboration, 2010.

\section{Marcantonio 2011}

Marcantonio ER. In the clinic: delirium. Annals of Internal Medicine 2011;154(11):TC6-1. [PUBMED: 21646553]

\section{Marcantonio 2012}

Marcantonio ER. Postoperative delirium: a 76-yearold woman with delirium following surgery. Journal of the American Medical Association 2012;308(1):73-81. [PUBMED: 22669559] 


\section{Marcantonio 2017}

Marcantonio ER. Delirium in hospitalized older adults. The New England Journal of Medicine 2017;377(15):1456-66. [PUBMED: 29298149]

\section{Marra 2017}

Marra A, Ely EW, Pandharipande PP, Patel MB. The ABCDEF Bundle in Critical Care. Critical Care Clinics 2017;33(2):225-43. [PUBMED: 28284292]

Marra 2017a

Marra A, Pandharipande PP, Patel MB. Intensive care unit delirium and intensive care unit-related posttraumatic stress disorder. The Surgical Clinics of North America 2017;97(6): 1215-35. [PUBMED: 29132506]

McCusker 2002

McCusker J, Cole M, Abrahamowicz M, Primeau F, Belzile E. Delirium predicts 12-month mortality. Archives of Internal Medicine 2002;162(4):457-63. [PUBMED: 11863480]

Morandi 2009

Morandi A, Jackson JC, Ely EW. Delirium in the intensive care unit. International Review of Psychiatry 2009;21(1): 43-58. [PUBMED: 19219712]

\section{Otter 2005}

Otter H, Martin J, Basell K, von Heymann C, Hein OV, Bollert $\mathrm{P}$, et al. Validity and reliability of the DDS for severity of delirium in the ICU. Neurocritical Care 2005;2 (2):150-8. [PUBMED: 16159057]

\section{Patel 2017}

Patel AK, Bell MJ, Traube C. Delirium in pediatric critical care. Pediatric Clinics of North America 2017;64(5): 1117-32. [PUBMED: 28941539]

\section{Pisani 2007}

Pisani MA, Murphy TE, Van Ness PH, Araujo KL, Inouye SK. Characteristics associated with delirium in older patients in a medical intensive care unit. Archives of Internal Medicine 2007;167(15):1629-34. [PUBMED: 17698685]

Reitsma 2005

Reitsma JB, Glas AS, Rutjes AW, Scholten RJ, Bossuyt $\mathrm{PM}$, Zwinderman $\mathrm{AH}$. Bivariate analysis of sensitivity and specificity produces informative summary measures in diagnostic reviews. Journal of Clinical Epidemiology 2005; 58(10):982-90. [PUBMED: 16168343]

Review Manager 2014 [Computer program] Nordic Cochrane Centre, The Cochrane Collaboration. Review Manager 5 (RevMan 5). Version 5.3. Copenhagen: Nordic Cochrane Centre, The Cochrane Collaboration, 2014.

Rutjes 2005

Rutjes AW, Reitsma JB, Vandenbroucke JP, Glas AS, Bossuyt PM. Case-control and two-gate designs in diagnostic accuracy studies. Clinical Chemistry 2005;51(8):1335-41. [PUBMED: 15961549]

\section{SCCM 2018}

Society of Critical Care Medicine. Critical Care Statistics. http://www.sccm.org/Communications/Critical-CareStatistics Date accessed: 05062018.

\section{Serafim 2015}

Serafim RB, Bozza FA, Soares M, do Brasil PE, Tura BR, Ely EW, et al. Pharmacologic prevention and treatment of delirium in intensive care patients: A systematic review. Journal of Critical Care 2015;30(4):799-807. [PUBMED: 25957498]

Shi 2013

Shi Q, Warren L, Saposnik G, Macdermid JC. Confusion assessment method: a systematic review and meta-analysis of diagnostic accuracy. Neuropsychiatric Disease and Treatment 2013;9:1359-70. [PUBMED: 24092976]

\section{Singh 2018}

Singh TD, O’Horo JC, Gajic O, Sakusic A, Day CN, Mandrekar J, et al. Risk factors and outcomes of critically ill patients with acute brain failure: A novel end point. Journal of Critical Care 2018;43:42-7. [PUBMED: 28843663]

Takwoingi Y 2015

Takwoingi Y, Guo B, Riley RD, Deeks JJ. Performance of methods for meta-analysis of diagnostic test accuracy with few studies or sparse data. Statistical Methods in Medical Research 2017;26(4):1896-911. [PUBMED: 26116616]

Thomason 2005

Thomason JW, Shintani A, Peterson JF, Pun BT, Jackson JC, Ely EW. Intensive care unit delirium is an independent predictor of longer hospital stay: a prospective analysis of 261 non-ventilated patients. Critical Care 2005;9(4): R375-81. [PUBMED: 16137350]

\section{Tobar 2010}

Tobar E, Romero C, Galleguillos T, Fuentes P, Cornejo $\mathrm{R}$, Lira MT, et al. Confusion Assessment Method for diagnosing delirium in ICU patients (CAM-ICU): cultural adaptation and validation of the Spanish version [Método para la evaluación de la confusión en la unidad de cuidados intensivos para el diagnóstico de delírium: adaptación cultural y validación de la versión en idioma español]. Medicina Intensiva 2010;34(1):4-13. [PUBMED: 19819041]

\section{Trogrlic 2015}

Trogrlic Z, van der Jagt M, Bakker J, Balas MC, Ely EW, van der Voort PH, et al. A systematic review of implementation strategies for assessment, prevention, and management of ICU delirium and their effect on clinical outcomes. Critical Care 2015;19:157. [PUBMED: 25888230]

\section{Trzepacz 1994}

Trzepacz PT. The neuropathogenesis of delirium. A need to focus our research. Psychosomatics 1994;35(4):374-91. [PUBMED: 7916159]

Van Rompaey 2009

Van Rompaey B, Elseviers MM, Schuurmans MJ, Shortridge-Baggett LM, Truijen S, Bossaert L. Risk factors for delirium in intensive care patients: a prospective cohort study. Critical Care 2009;13(3):R77. [PUBMED: 19457226]

\section{Whiting 2011}

Whiting P, Westwood M, Beynon R, Burke M, Sterne JA, Glanville J. Inclusion of methodological filters in 
searches for diagnostic test accuracy studies misses relevant studies. Journal of Clinical Epidemiology 2011;64(6):602-7. [PUBMED: 21075596 ]

\section{Whiting 2011a}

Whiting PF, Rutjes AW, Westwood ME, Mallett S, Deeks JJ, Reitsma JB, et al. QUADAS-2: a revised tool for the quality assessment of diagnostic accuracy studies. Annals of Internal Medicine 2011;155(8):529-36. [PUBMED: 22007046]

\section{Witlox 2010}

Witlox J, Eurelings LS, de Jonghe JF, Kalisvaart KJ,
Eikelenboom P, van Gool WA. Delirium in elderly patients and the risk of postdischarge mortality, institutionalization, and dementia: a meta-analysis. Journal of the American Medical Association 2010;304(4):443-51. [PUBMED: 20664045]

\section{Wong 2010}

Wong CL, Holroyd-Leduc J, Simel DL, Straus SE. Does this patient have delirium?: value of bedside instruments. Journal of the American Medical Association 2010;304(7): 779-86. [PUBMED: 20716741]

* Indicates the major publication for the study

\section{A P P E N D I C E S}

\section{Appendix I. MEDLINE search strategy}

1. exp Delirium/

2. deliri*.mp.

3. "acute confusion*".ti,ab.

4. "acute organic psychosyndrome”.ti,ab.

5. "acute brain syndrome".ti,ab.

6. "metabolic encephalopathy".ti,ab.

7. "acute psycho-organic syndrome”.ti,ab.

8. "clouded state".ti,ab.

9. "clouding of consciousness".ti,ab.

10. “exogenous psychosis”.ti,ab.

11. "toxic psychosis".ti,ab.

12. "toxic confusion".ti,ab.

13. Delirium, Dementia, Amnestic, Cognitive Disorders/su [Surgery]

14. obnubilat*.ti,ab.

15. or/1-14

16. "confusion assessment method".ti,ab.

17. CAM-ICU*.ti,ab.

18. "CAM-FAM*”.ti,ab.

19. ("mCAM-ED” or "CAM-ED”).ti,ab.

20. (CAM adj3 (intensive or deliri* or ICU)).ti,ab.

21. or/ $16-20$

22. 15 and 21

23. reproducibility.ti,ab.

24. diagnos*.ti.

25. sensitivit*.ab.

26. specificit*.ab.

27. (ROC or "receiver operat").ab.

28. "Reproducibility of Results"/

29. ("Area under curve" or AUC).ab.

30. sROC.ab.

31. accura*.ti,ab.

32. (likelihood adj3 (ratio* or function*)).ab.

33. ((true or false) adj3 (positive* or negative*)).ab.

Confusion Assessment Method for the intensive care unit (CAM-ICU) for the diagnosis of delirium in adults in critical care settings 
34. ((positive* or negative* or false or true) adj3 rate* $^{*}$.ti,ab.

35. "Sensitivity and Specificity"/

36. or/23-35

37. exp *Delirium/di [Diagnosis]

38. (delirium adj3 (detect* or identify* or diagnos* or assess*)).ti,ab.

39. 37 or 38

40.36 and 39

41.22 or 40

\section{Appendix 2. Data Extraction Pro-forma}

\section{STUDY IDENTIFICATION AND STUDY TYPE}

\section{DETAILS}

ID (Author, year)

Authors

Journal

Country in which study is conducted

Period of data collection

Objective

Study design (select one)

o Cross sectional test accuracy study

o Cohort test accuracy study

o Comparison of the accuracy of tests or testing strategies in two different populations

o Any other study where estimation of test accuracy was not the primary objective
Subtype of delirium, if provided
o Hyperactive
o Hypoactive
o Mixed
o Not stated

Severity of delirium, if provided

Duration of symptoms, if provided

\section{PATIENT SELECTION}




\section{A. DETAILS}

Describe methods of patient selection (cut and paste from paper if possible)

Describe characteristics included patients; previous testing, presentation, intended use of index test, and setting (cut and paste from paper if possible)

Number of participants

\begin{tabular}{ll}
\hline Setting & o Medical ICU \\
& o Surgical ICU \\
& o Mixed ICU \\
& o Other ICUs \\
\hline
\end{tabular}

Eligibility criteria

Exclusion criteria

Age

Gender

Co-morbidities

Type of ventilatory assistance

o Mechanical

o Spontaneous

\section{B. ASSESSMENT RISK OF BIAS}

Was a consecutive or random sample of patients enrolled?

o "Yes" if it is well described in the paper that a consecutive or random sample of consecutive patients admitted to the intensive care unit were enrolled

o "No" if the sample was non-random or ICU patients were not consecutively recruited

o "Unclear" if there is insufficient information to make a judgment on the selection of patients

Was a case control design avoided?

o "Yes" = The study used other research design (such as crosssectional and cohort)

o "No" = The study should be excluded for this review (see eligibility criteria)

o Unclear $=$ if there is insufficient information to make a judgment on the design

Did the study avoid inappropriate inclusions?

o "Yes" if inclusion and exclusion criteria clearly described and appropriate

o "No" if inclusion and exclusion criteria clear but include inap-

Confusion Assessment Method for the intensive care unit (CAM-ICU) for the diagnosis of delirium in adults in critical care settings

(Protocol)

Copyright $\odot 2018$ The Cochrane Collaboration. Published by John Wiley \& Sons, Ltd. 
propriate subjects, such as patients admitted in non-critical units, pediatric population or patients with history of severe dementia, psychosis, or neurologic disease that would confound the diagnosis of delirium

o Unclear" if there is insufficient information to make a judgment on the inclusion/exclusion of subjects

Could the selection of patients have introduced bias?

o "High" if it is clear that bias is introduced through, for example, non-random selection

o "Low" if the selection of patients is clearly described and does not introduce bias

o "Unclear" if there is insufficient information to make a judgment on the impact of selection on bias

\section{CONCERNS ABOUT APLICABILITY}

Are there concerns that included patients do not match the review question? o "High" if included patients are inherently different from the cohort of patients who would be expected to receive the CAMICU

o "Low" if there are no such concerns.

o "Unclear" if patient characteristics are not sufficiently clearly explained to make a judgment on patient inclusion

\section{INDEX TEST}

\section{A. DETAILS}

Describe the index test and how it was conducted and interpreted (cut and paste from paper if possible)

Frequency of testing

Operator characteristics (e.g. training)

Thresholds/Criteria used to define positive and negative tests for target condition

\section{B. ASSESSMENT RISK OF BIAS}

Were the index test results interpreted without knowledge of the results of the reference standard? o "Yes" if the paper states that the CAM-ICU is interpreted by clinician/ researcher who did not know the results of the reference standard

o "No" if the results of the index test were known by the clinicians performing the reference test, or if the same clinician/researcher performed both tests

o "Unclear" if there is insufficient information to make a judgment 
If a threshold was used, was it prespecified? o "Yes" if the research used a pre-specified criteria of positivity (Feature 1 plus 2 and either 3 or 4 present = CAM-ICU positive= delirium present)

o "Unclear" if there is no clear the CAM-ICU positive criteria used

o "No" if there are explorations of positivity criteria in the data analysis

Could the conduct or interpretation of the index test have introduced bias? o "High" if a subset of CAM-ICU tests were conducted or interpreted in a different manner, or under different conditions, or by people with differing levels of training

o "Low" if it is clear that the conduct and interpretation of CAMICU was appropriate and could not have introduced bias

o "Unclear" if there is insufficient information presented to assess the potential of conduct and interpretation of the CAM-ICU to introduce bias

\section{CONCERNS ABOUT APLICABILITY}

Are there concerns that the index test, its conduct, or interpretation differ from the review question? o "Yes" if the index test is not the CAM-ICU for diagnosis of delirium, or if the conduct of test or its interpretation is not applicable to the review question

o "No" if there are no concerns based on the information presented o "Unclear" if the conduct of test or its interpretation are not sufficiently clearly explained to make a judgment on this issue

\section{REFERENCE STANDARD}

\section{A. DETAILS}

Describe the reference standard and how it was conducted and interpreted (cut and paste from paper if possible)

Operator characteristics (e.g. training)

Thresholds/Criteria used to define positive and negative tests for target condition, if provided

\section{B. ASSESSMENT RISK OF BIAS}

Is the reference standard likely to correctly classify the target condition? o "Yes" if the reference standard used in the paper matches those chosen in this protocol (DSM criteria applied by a expert)

o "No" if the above criteria is not met.

o "Unclear" if insufficient information is presented. 
Were the reference standard results interpreted without knowledge of the results of the index test? o "Yes" if the paper states that the reference test is interpreted by clinicians/researchers who had not seen the reference standard results

o "No" if the result(s) of the CAM-ICU were known to the individual performing the DSM criteria

o "Unclear" if there is insufficient information to make a judgment about this issue

Could the reference standard, its conduct, or its interpretation have introduced bias?

o "High" if a subset of reference standard tests were conducted or interpreted in a different manner, or under different conditions o "Low" if it is clear that the conduct and interpretation of all reference standard tests were appropriate and could not have introduced bias

o "Unclear" if there is insufficient information presented to assess the potential of conduct and interpretation of the reference standard test to introduce bias

\section{CONCERNS ABOUT APLICABILITY}

Are there concerns that the target condition as defined by the o "High" if the target condition is not delirium or it is not clearly reference standard does not match the review question?

stated

o "Low" if it is clearly stated that the target condition is delirium

o "Unclear" if there is insufficient information presented to assess potential concerns about the target condition

\section{FLOW AND TIMING}

\section{A. DETAILS}

Describe any patients who did not receive the index tests or reference standard or who were excluded from the $2 \times 2$ table (refer to flow diagram)

Describe the interval and any interventions between index tests and the reference standard

\section{B. ASSESSMENT RISK OF BIAS}

Was there an appropriate interval between index test(s) and reference standard? o "Yes" if the time between CAM-ICU administration and reference standard was less than 24 hours

o "No" if the time is longer than 24 hours for a significant proportion of patients

o "Unclear" " if there is insufficient information about the interval between the CAM-ICU and the reference standard 
Did all patients receive a reference standard?

Were all patients included in the analysis?
Did all patients receive the same reference standard?

o "Yes" if the same reference standard was used for all patients

o "No" if different reference standards were used.

o "Unclear" if this cannot be determined from the information presented in the paper

o "Yes" if the same reference standard was used for all patients o "No" if different reference standards were used.

o "Unclear" if this cannot be determined from the information presented in the paper

o "Yes" if there were no withdrawals or exclusions, or if those reasons are adequately explained (e.g. with a flow chart)

o "No" if withdrawals or exclusions are not explained or accounted for

o "Unclear" if withdrawals or exclusions cannot be determined or if there is insufficient information to judge this

Could the patient flow have introduced bias?

o "High" if subsets of patients or samples were treated, included or excluded in systematic ways, which could have introduced bias o "Low" if patient flow is reported clearly and does not have the potential to introduce significant bias

o "Unclear" if there is insufficient information presented to assess potential concerns about the patient flow

\section{REFERENCE STANDARD REFERENCE STANDARD POSITIVE NEGATIVE}

\begin{tabular}{|c|c|c|c|}
\hline INDEX TEST POSITIVE & $\mathrm{TP}(\mathrm{n} / \%=):$ & $\mathrm{FP}(\mathrm{n} / \%=)$ : & $\begin{array}{l}\text { TOTAL INDEX TEST POSI- } \\
\text { TIVE (n/\%=): }\end{array}$ \\
\hline INDEX TEST NEGATIVE & $\mathrm{FN}(\mathrm{n} / \%=)$ & $\mathrm{TN}(\mathrm{n} / \%=):$ & $\begin{array}{l}\text { TOTAL INDEX TEST NEGA- } \\
\text { TIVE (n/\%=): }\end{array}$ \\
\hline PREVALENCE (n/\%=): & SENSITIVITY: & SPECIFICITY: & TOTAL: \\
\hline
\end{tabular}




\section{Appendix 3. Anchoring statements for quality assessment of CAM-ICU in diagnosis of delirium}

\begin{tabular}{l|l}
\hline Patient Selection & \\
\hline Was a consecutive or random sample of patients enrolled? & $\begin{array}{l}\text { "Yes" if it is well described in the paper that consecutive patients } \\
\text { with suspected delirium were enrolled. } \\
\text { "No" if the sample was non-random or ICU patients were not } \\
\text { consecutively recruited } \\
\text { "Unclear" if there is insufficient information to make a judgment } \\
\text { on the selection of patients }\end{array}$ \\
\hline
\end{tabular}

Was a case control design avoided?

"Yes"= The study used other research design (such as cross-sectional and cohort).

"No" = The study should be excluded for this review (see eligibility criteria).

Unclear $=$ if there is insufficient information to make a judgment on the design

Did the study avoid inappropriate inclusions?

"Yes" if inclusion and exclusion criteria clearly described and appropriate.

"No" if inclusion and exclusion criteria clear but include inappropriate subjects, such as patients admitted in non-critical units, pediatric population or patients with history of severe dementia, psychosis, or neurologic disease that would confound the diagnosis of delirium.

"Unclear" if there is insufficient information to make a judgment on the inclusion/exclusion of subjects

Could the selection of patients have introduced bias?

"High" if it is clear that bias is introduced through, for example, non-random selection.

"Low" if the selection of patients is clearly described and does not introduce bias.

"Unclear" if there is insufficient information to make a judgment on the impact of selection on bias

Are there concerns that included patients do not match the review question?

"High" if included patients are inherently different from the cohort of patients who would be expected to receive the CAM-ICU. "Low" if there are no such concerns.

"Unclear" if patient characteristics are not sufficiently clearly explained to make a judgment on patient inclusion

Index Test

Were the index test results interpreted without knowledge of the results of the reference standard?

"Yes" if the paper states that the CAM-ICU is interpreted by clinician/ researcher who did not know the results of the reference standard.

"No" if the results of the index test were known by the clinicians performing the reference test, or if the same clinician/researcher performed both tests.

"Unclear" if there is insufficient information to make a judgment

Confusion Assessment Method for the intensive care unit (CAM-ICU) for the diagnosis of delirium in adults in critical care settings

(Protocol)

Copyright @ 2018 The Cochrane Collaboration. Published by John Wiley \& Sons, Ltd. 

ture 1 plus 2 and either 3 or 4 present = CAM-ICU positive= delirium present).

"Unclear" if there is no clear the CAM-ICU positive criteria used. "No" if there are explorations of positivity criteria in the data analysis

Could the conduct or interpretation of the index test have introduced bias?
"High" if a subset of CAM-ICU tests were conducted or interpreted in a different manner, or under different conditions, or by people with differing levels of training.

"Low" if it is clear that the conduct and interpretation of CAMICU was appropriate and could not have introduced bias.

"Unclear" if there is insufficient information presented to assess the potential of conduct and interpretation of the CAM-ICU to introduce bias

Are there concerns that the index test, its conduct, or interpretation differ from the review question?

"Yes" if the index test is not the CAM-ICU for diagnosis of delirium, or if the conduct of test or its interpretation is not applicable to the review question.

"No" if there are no concerns based on the information presented. "Unclear" if the conduct of test or its interpretation are not sufficiently clearly explained to make a judgment on this issue

\section{Reference Standard}

Is the reference standard likely to correctly classify the target condition? "Yes" if the reference standard used in the paper matches those chosen in this protocol (DSM criteria applied by a expert)

"No" if the above criteria is not met.

"Unclear" if insufficient information is presented.

Were the reference standard results interpreted without knowledge of "Yes" if the paper states that the reference test is interpreted by the results of the index test? clinicians/researchers who had not seen the reference standard results.

"No" if the result(s) of the CAM-ICU were known to the individual performing the DSM criteria.

"Unclear" if there is insufficient information to make a judgment about this issue

Could the reference standard, its conduct, or its interpretation have introduced bias?

"High" if a subset of reference standard tests were conducted or interpreted in a different manner, or under different conditions. "Low" if it is clear that the conduct and interpretation of all reference standard tests were appropriate and could not have introduced bias.

"Unclear" if there is insufficient information presented to assess the potential of conduct and interpretation of the reference standard test to introduce bias 
Are there concerns that the target condition as defined by the reference standard does not match the review question?
"High" if the target condition is not delirium or it is not clearly stated.

"Low" if it is clearly stated that the target condition is delirium.

"Unclear" if there is insufficient information presented to assess potential concerns about the target condition

\section{Flow and timing}

Was there an appropriate interval between index test(s) and reference standard?

Did all patients receive a reference standard?

Did all patients receive the same reference standard?

Could the patient flow have introduced bias?

\section{Were all patients included in the analysis?}

"Yes" if the time between CAM-ICU administration and reference standard was less than 24 hours.

"No" if the time is longer than 24 hours for a significant proportion of patients.

"Unclear" " if there is insufficient information about the interval between the CAM-ICU and the reference standard

"Yes" if the same reference standard was used for all patients.

"No" if different reference standards were used.

"Unclear" if this cannot be determined from the information presented in the paper

"Yes" if the same reference standard was used for all patients "No" if different reference standards were used.

"Unclear" if this cannot be determined from the information presented in the paper

"Yes" if there were no withdrawals or exclusions, or if those reasons are adequately explained (e.g. with a flow chart).

"No" if withdrawals or exclusions are not explained or accounted for.

"Unclear" if withdrawals or exclusions cannot be determined or if there is insufficient information to judge this

"High" if subsets of patients or samples were treated, included or excluded in systematic ways, which could have introduced bias. "Low" if patient flow is reported clearly and does not have the potential to introduce significant bias.

"Unclear" if there is insufficient information presented to assess potential concerns about the patient flow 


\section{CONTRIBUTIONS OFAUTHORS}

Draft the protocol: FM, IA, PS, MNP, JZ, TQ

Develop and run the search strategy: FM, PS

Obtain copies of studies: FM

Select which studies to include (2 people): FM, GD, FG

Extract data from studies (2 people): FM, GD, FG

Enter data into RevMan: FM, PS

Carry out the analysis: FM, IA, MNP, JZ, PS

Interpret the analysis: FM, IA, MNP, JZ, PS, TQ

Draft the final review: FM, IA, PS, MNP, JZ, FM, GD, FG, TQ

Update the review: FM, PS, GD, FG, IA, TQ

\section{DECLARATIONSOF INTEREST}

Fabián Miranda has no conflicts of interest to declare.

Ingrid Arevalo-Rodriguez has no conflicts of interest to declare.

Gonzalo Díaz has no conflicts of interest to declare.

Francisco González has no conflicts of interest to declare.

Maria N Plana has no conflicts of interest to declare.

Javier Zamora has no conflicts of interest to declare.

Pamela Seron has no conflicts of interest to declare.

Terry Quinn has no conflicts of interest to declare.

\section{SOURCES OF SUPPORT}

\section{Internal sources}

- No sources of support supplied

\section{External sources}

- NIHR, UK.

This protocol was supported by the National Institute for Health Research (NIHR), via Cochrane Infrastructure funding to the Cochrane Dementia and Cognitive Improvement group. The views and opinions expressed therein are those of the authors and do not necessarily reflect those of the Systematic Reviews Programme, NIHR, National Health Service or the Department of Health 